\title{
An Experimental Study and Concept Evaluation on Tree-Interior Imaging Radar Using Sinusoidal Template-Based Focusing Algorithm
}

\author{
Betül Yılmaz, Serhat Gökkan, and Caner Özdemir \\ Department of Electrical and Electronics Engineering, Mersin University, Ciftlikkoy 33343, Mersin, Turkey \\ Correspondence should be addressed to Serhat Gökkan; sgokkan@mersin.edu.tr
}

Received 6 June 2017; Accepted 14 September 2017; Published 1 November 2017

Academic Editor: Ping Feng Pai

Copyright (c) 2017 Betül Yılmaz et al. This is an open access article distributed under the Creative Commons Attribution License, which permits unrestricted use, distribution, and reproduction in any medium, provided the original work is properly cited.

\begin{abstract}
An algorithm for detecting cavities inside a tree-body is presented with simulation and measured examples. The details of the imaging algorithm that is based on sinusoidal template focusing routine are given. First, the algorithm is tested with the simulation scenario for which perfect reconstruction of the simulated cavity structure together with tree-body is successfully formed in MATLAB programming environment. Then, the algorithm is applied to the measurement data that have been collected from a laboratory set-up. Collected backscattered measurements from the tree-body (with cavity) structure are used to generate the image of the scene by the help of our proposed algorithm. The resultant radar images of the measured data collected from the laboratory arrangement have shown the applicability of the developed algorithm for the detection of cavity structures inside tree-bodies.
\end{abstract}

\section{Introduction}

The detection of voids or cavities within tree-bodies at microwave band has become an emerging technology for the determination of unhealthy and weak trees $[1,2]$. Every year tens of people are being killed all around the world due to falling tree incidents and related incidents [1-4]. Although most of the trees seem to be healthy and strong when looking from the outside, it is not easy to realize the existence of interior cavities that might occur due to fungus and worm attacks by using conventional methods such as acoustic imaging techniques [5], resistivity measurement methods [6], and thermographic inspection [7]. It is known by the experts that decaying wood is expected to have a different moisture content and density; therefore, this part of the treebody should have different electrical characteristics such as dielectric constant and conductivity [8]. One of the most applied methods is the ultrasonic tomography that can sense decay structures only under special circumstances [9]. This technique uses the mechanical behavior of wood that usually depicts a strong anisotropy such that ultrasonic pulses are being used to sense this discontinuity caused by the cavity. On the other hand, ultrasonic pulses in wood are usually strongly attenuated; therefore, this method requires high signal sensitivity and dynamic range while acquiring and processing the raw data $[10,11]$. Recently, using obstaclepenetrating radar technologies to detect and image cavities within a tree-body has been increasingly gaining attention thanks to recent advances in radar hardware and radar signal processing techniques [12-15]. It is eminent by the electromagnetic (EM) community that microwave imaging techniques have the advantages of providing high resolution features and requiring relatively smaller amount of power to penetrate such obstacles. Algorithms used in groundpenetrating-radar (GPR) [16-18] and through-wall-imagingradar (TWIR) [19-21] seem to provide promising solutions to this special problem. Also, circular Radon transform could be an opportunity to focus the scattered electric field data collected from a circular measurement set-up for a different application [22].

In this work, we offer a practical but also a fast focusing/migration method that can be readily used for treeinterior imaging radar (TIIR) applications. We are presenting a conceptual study on TIIR by suggesting an imaging/focusing method that has been specially tailored to 


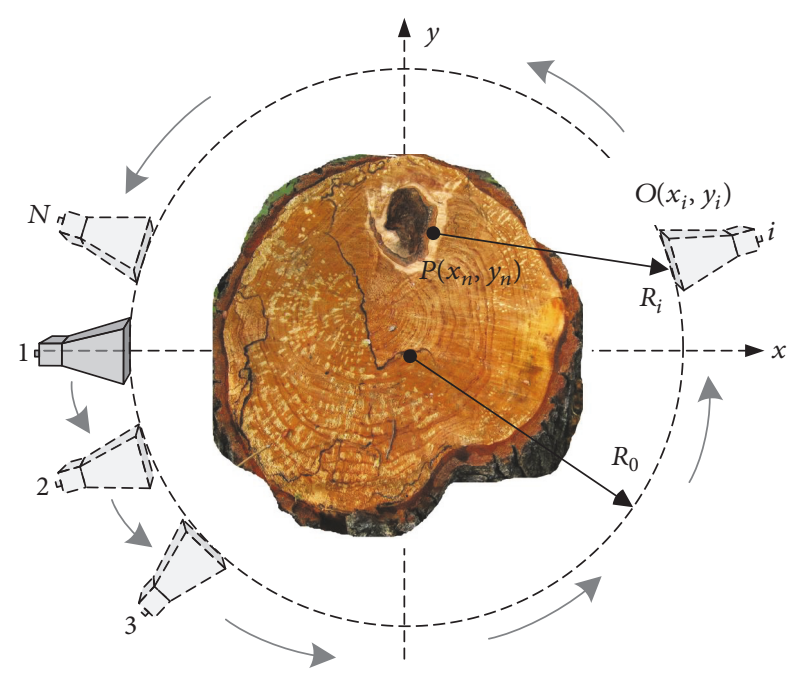

FIgURE 1: The geometry of TIIR problem.

this specific application and also demonstrating a proof-ofconcept study by providing and assessing the results of related simulations and measurements from a test-bed.

The paper is organized as follows: in the next section, we present the steps and the details of our imaging algorithm for the TIIR problem. In the third section, the validity of the proposed method is first tested with the help of simulated data that are generated with point scatterer model. In the following section, a measurement set-up for TIIR experiments is constructed and both the simulation of the experimental set-up and measurements conducted with real tree-body with cavity are presented. The results after applying the proposed methods are provided. Resultant radar images of both the simulation and the measurement data validate the effectiveness and the success of our suggested algorithm. In the final section, the work is concluded by discussing the applicability and assessment of the measurement system with the proposed algorithm.

\section{Imaging/Focusing Algorithm for TIPR}

The geometry of our TIIR problem can be described as illustrated in Figure 1 where the tree-body is circularly scanned by a directive antenna for a total of $M$ distinct lookaspects covering the whole azimuth angles. The radius of the scanning circle is $R_{0}$. The frequency-diverse backscattered electric field data are collected in the monostatic configuration of the radar. For any point scatterer, $P\left(x_{n}, y_{n}\right)$ was assumed to be on the perimeter of the cavity within the radius $R_{0}$; therefore, one-dimensional (1D) electric field data can be obtained by the antenna of the $i_{\text {th }}$ position as

$$
\mathbf{E}_{\mathbf{i}}^{\mathbf{s}}(\mathbf{k})=A_{i} \exp \left(-j 2 \mathbf{k} r_{i}\right)
$$

Here, $r_{i}=\operatorname{sqrt}\left[\left(x_{n}-x_{i}\right)^{2}+\left(y_{n}-y_{i}\right)^{2}\right]$ is the distance between the scatterer and the antenna and $\mathbf{k}$ is the wavenumber vector. This result is valid for the following assumptions: first, we assume isotropic radiation of the electromagnetic (EM) wave inside the tree-body such that the EM wave travels along its propagation direction. This assumption is usually true for the decayed trees since such trees usually dry in the inside due to decaying phenomenon. It is reported that the dielectric constant of dried wood material is on the order of 1.4 to 2.9 in practice [23]. Therefore, the moisture content for the decayed trees is usually very low and does not present significant anisotropy in terms of the water content of the tree-body. This situation also helps radar signal to penetrate the tree-body better since the relative electric permittivity contrast between the air and the tree structure is not high. Secondly, the scattering structures within the tree-body are assumed to be represented with point scatterer model that is very effective and commonly used in many radar applications [24]. Therefore, near field boundary conditions for the air void and the tree-body are not imposed. Although such modelling will provide a more realistic representation, the surface modelling of air void would be quite complex and yield a very complex calculation of the EM reflection. Therefore, we have encountered a more basic and simplified EM scattering calculation model to ease the numerical load of the problem. Furthermore, such kind of study is out of this research work. Yet, modelling boundary points such as surface of cavities with perfect point scatters has proven to be an effective way of calculating the scattering from such boundaries in many radar problems $[25,26]$. Thirdly, any multipath phenomenon is not accounted as all radar imaging algorithms are based on only single-reflection assumption.

Based on the above assumptions, then, (1) can be rewritten as a summation over scatterer and provides a twodimensional (2D) data by considering different look angles as the following for a finite number of point scatterers $(M)$ that form the tree-body:

$$
\mathbf{E}^{\mathbf{s}}(\mathbf{k}, \boldsymbol{\phi})=\sum_{i=1}^{M} A_{i} \exp \left(-j 2 \mathbf{k} \mathbf{R}_{\mathbf{i}}\right)
$$

where $\mathbf{R}_{\mathbf{i}}=\operatorname{sqrt}\left[\left(\mathbf{X}-x_{i}\right)^{2}+\left(\mathbf{Y}-y_{i}\right)^{2}\right]$ is the distance vector between the scatterer and all the positions of radar for whole azimuth observation angles of $\phi$ ranging from 1 to $M$ and $\mathbf{k}$ is the wave number for the stepped frequencies for a total of $N$ distinct frequencies. Then, $\mathbf{X}$ and $\mathbf{Y}$ vectors correspond to Cartesian coordinates of $M$ antenna positions, accordingly as given below:

$$
\begin{aligned}
& \mathbf{X}=R_{0} \cos \phi, \\
& \mathbf{Y}=R_{0} \sin \phi .
\end{aligned}
$$

Taking the one-dimensional (1D) inverse Fourier transform (IFT) of (2) along the spatial frequency axis, one can easily get the $2 \mathrm{D}$ range-angle data, $E^{\mathcal{s}}(\mathbf{r}, \phi)$. In this resultant rangeangle data, any point $P\left(x_{n}, y_{n}\right)$ exhibits a sinusoidal behavior for the $360^{\circ}$ variation of azimuth angle $\phi$ because of circular scanning arrangement.

Our detection and imaging algorithm can be briefly summarized as follows: (i) after obtaining the $2 \mathrm{D}$ range-angle backscattered electric field data, $\mathbf{E}^{\mathbf{s}}(\mathbf{r}, \phi)$, we select an image window in 2D (range, angle) domain that covers the whole geometry. It is important to note that the range-extent of the 


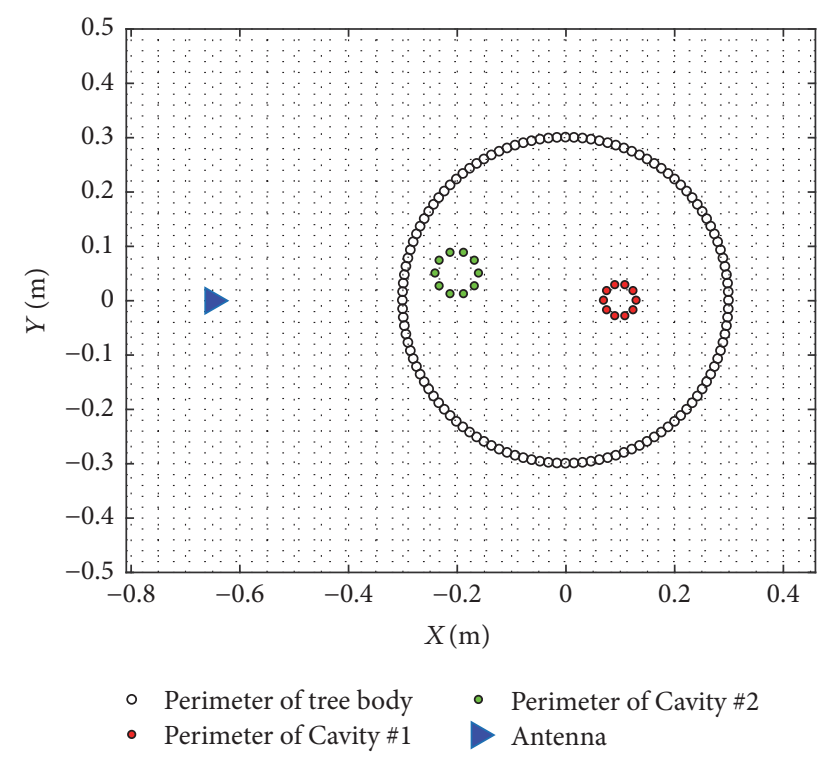

FIgURE 2: Simulation geometry for the TIIR algorithm.

image window should be larger than or at least equal to the diameter of scanning, that is, $2 R_{0}$. (ii) Next, we iteratively pick all the points; $P\left(x_{p}, y_{p}\right)$ within the selected window. (iii) Then, we form a template vector for each look angle of $\phi_{i}$ by writing the following template equation:

$$
\mathbf{R}_{\text {temp }}^{\mathbf{n}}\left(\phi_{\mathbf{n}}\right)=\operatorname{sqrt}\left[\left(\mathbf{X}-x_{p}\right)^{2}+\left(\mathbf{Y}-y_{p}\right)^{2}\right] .
$$

(iv) Afterwards, we go back to $2 \mathrm{D} E^{s}(\mathbf{r}, \phi)$ data and pick the data under $\left(\mathbf{R}_{\text {temp }}^{\mathbf{n}}, \phi_{\mathbf{n}}\right)$ points. At this point, a precise data interpolation scheme needs to be employed to lessen the error associated with data interpolation. (v) In the final step, we add all the data under this template vector and record the result in a new $2 \mathrm{D}$ image matrix at $\left(x_{p}, y_{p}\right)$ location. The new, reconstructed image data at $\left(x_{p}, y_{p}\right)$ location becomes a pixel in the final image. The algorithm successively continues until all the points in the selected image window are passed through the algorithm. The resultant $\mathbf{I}(x, y)$ matrix is the focused image.

\section{Simulation Results}

The above-explained algorithm was first tested by a simulation scenario whose illustration is given in Figure 2. The simulation scene was constructed in MATLAB programming environment [27] with the assumptions that were listed in the previous section. To be able to test and evaluate the performance of this technique with ideal conditions, perimeter of the tree-body (shown as black dots) was assumed to be composed of a total of 60 perfect point scatterers with the identical reflectivity amplitudes of " 1 ," that is, independent of frequency and aspect. The radius of the tree-body is taken as $60 \mathrm{~cm}$. In the simulation, there were two cavity regions; one was centered at $(10 \mathrm{~cm}, 0)$ while the other one was located at $(-20 \mathrm{~cm}, 5 \mathrm{~cm})$. Both cavities were considered to be in the form of circles with diameters of $8 \mathrm{~cm}$ and $6 \mathrm{~cm}$ for the first and second void regions, respectively. The circumference of the cavities was also assumed to be composed of perfect point scatterers with equal EM reflectivities of " 0.5 " that are independent of frequency and angle. Dielectric constant for the tree-body was taken as 1.65 and assumed to be homogeneous within the tree-body. The radar antenna was positioned at $35 \mathrm{~cm}$ away from the tree's surface and moved along a circular track for a total of 360 distinct look angles to collect the backscattered data. For each of the look angles, frequency-diverse backscattered electric field was gathered for the stepped frequencies ranging from 1 to $8 \mathrm{GHz}$ for the total of 100 discrete frequency points. Therefore, the backscattered electric field data were collected on the $2 \mathrm{D}$ frequency-angle plane such that a matrix of $100 \times 360$ points was obtained.

We apply the steps of our focusing algorithm that was based on summation of the data beneath the sinusoidal template. In Figure 3(a), the range profile matrix for different look angles, $\mathbf{E}^{\mathbf{s}}(\mathbf{r}, \phi)$, was obtained. We have several observations about the range-angle raw data in Figure 3(a) as follows: (i) the scattering from the nearest point from the circular tree-body geometry shows up as a straight line since both the scanning path and the tree-body are in the form of circles. This line occurred at a range distance of $35 \mathrm{~cm}$ as expected. (ii) Scatterers on the perimeter of both cavities come out as sinusoids of different oscillating amplitudes due to different round-trip distances between the antenna and the scatterers due to circular movement of the radar while collecting the data. In fact, the peak amplitudes of these oscillating sinusoids depend on their distances from the center of rotation. For our simulation example, these numbers are $4 \mathrm{~cm}$ and $3 \mathrm{~cm}$ for the first and the second cavity, respectively. The range-angle image in Figure 3(a) exactly exhibits this behavior as we have anticipated. (iii) The widths of the sinusoids represent the diameters of the cavities. From the figure, the widths of the sinusoidal behavior are $8 \mathrm{~cm}$ and $6 \mathrm{~cm}$ that are precisely the same values of real diameters for the cavity regions. (iv) The starting point of the sinusoidal behavior actually pinpoints the exact location of the cavity: for example, if the sinusoids start from the maximum value, the cavity is located at the nearest point to the antenna's location. If the sinusoid starts from the minimum value, then, the cavity is at $180^{\circ}$ away from the antenna. (v) Center point of the scanning will show up as a straight line in the rangeangle image. Therefore, if there is a small cavity at the center, its image will be a horizontal line at the half wave between the scatterings from near and far points of the tree-body. (vi) Second straight line around $95 \mathrm{~cm}$ in range-angle image in Figure 3(a) corresponds to scattering from far-most point of tree-body. As the radar moves around a circle, the distance from the far-most point of the tree-body stays constant at $95 \mathrm{~cm}$ as it was obtained in Figure 3(a). After analyzing the range-angle image in Figure 3(a) that certainly tells us many facts, we applied the steps of the algorithm such that the final focused image $\mathbf{I}(x, y)$ was obtained as given in Figure 3(b). As it is clearly seen from the figure, both the tree-body and the cavity regions were successfully imaged in the resultant focused image at their correct locations in the Cartesian coordinates. Of course, the image strength of cavities is much 


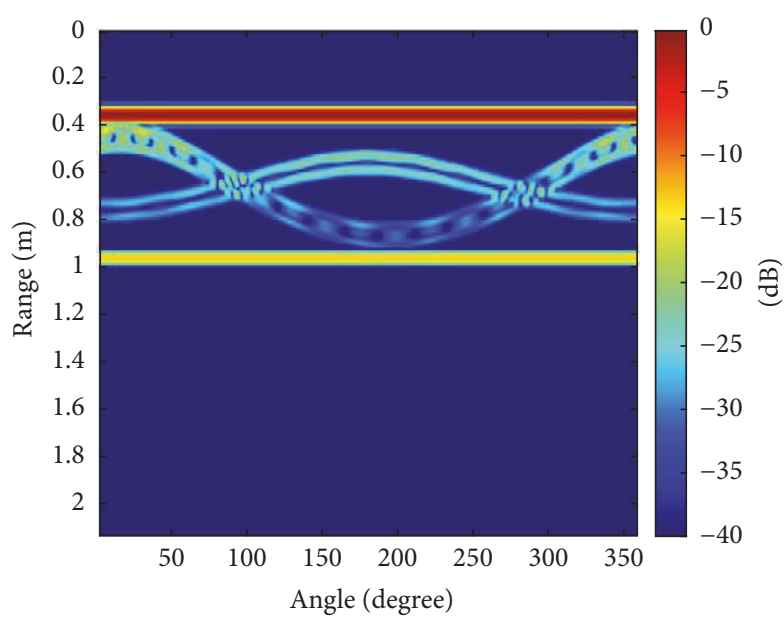

(a)

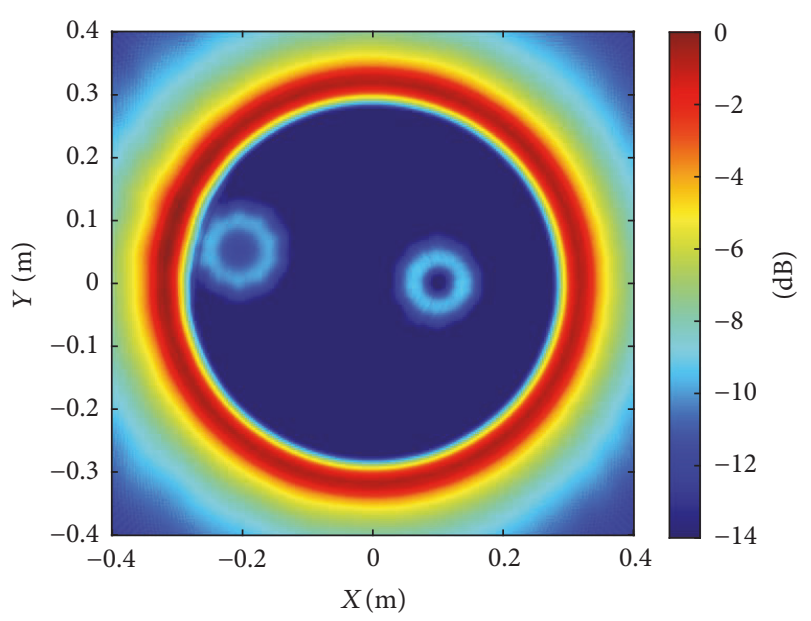

(b)

Figure 3: Simulation results for the geometry in Figure 2: (a) range-angle image; $\mathbf{E}^{\mathbf{s}}(r, \phi)$, (b) focused image; $\mathbf{I}(x, y)$ after applying our algorithm.

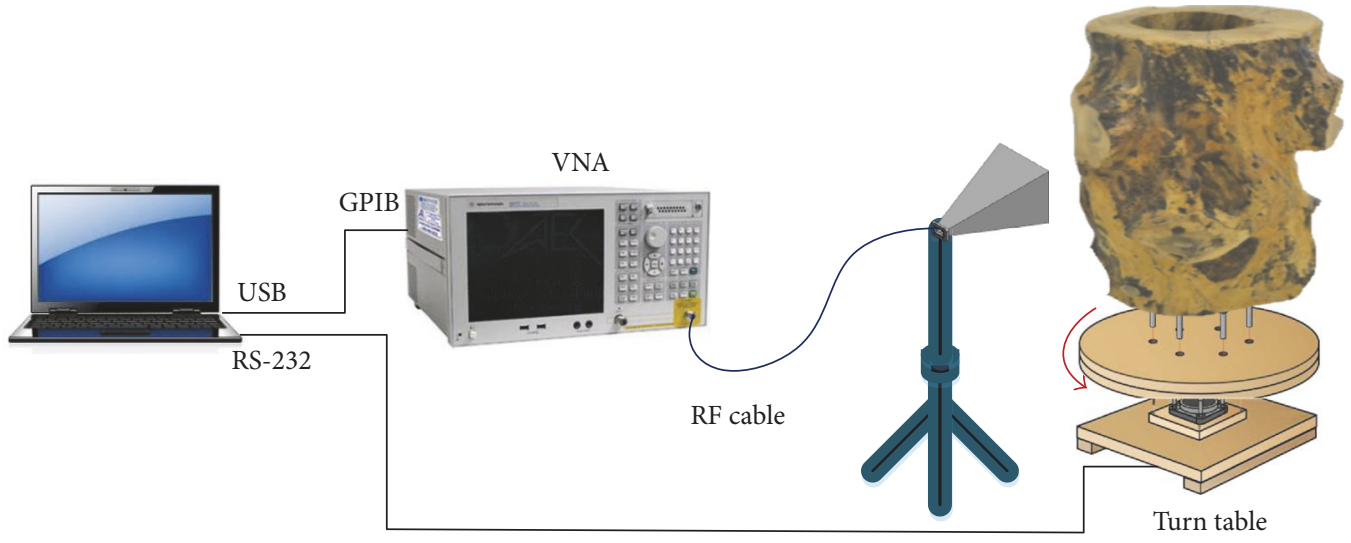

FIGURE 4: The measurement set-up for the TIIR experiment.

smaller than that of tree's surface due to decaying of EM wave with $1 /\left(4 \pi R^{2}\right)$ term.

The fidelity of the resultant image in our simulation is compared to other methods such as Radon transform [21] and ultrasonic techniques are as follows [9]: focused image can be obtained from Radon transform simulation method that uses projection data associated with cross-sections of an object but this method requires complex calculations for obtaining the final image. Therefore, its implementation is more difficult and takes much more time. Ultrasonic simulation method also can be used for imaging but the disadvantage of this method is the weakness of the ultrasonic signals to be able to penetrate the tree-body. To ensure the required minimum detectable signal level, many receivers are usually required along the perimeter of tree-body. Our method is more practical and has faster migration method to be applied for TIIR applications.

\section{Measurement Set-Up and Experimental Results}

To assess the effectiveness of our TIIR detection/imaging algorithm with real experimental data, we have constructed a measurement test-bed at our anechoic chamber of Mersin University's Advanced Technologies Research Center (MEATRC) facility. The geometry of the measurement setup is shown in Figure 4. In this set-up, a Vector Network Analyzer (VNA) [Agilent ENA5071B] was utilized to generate/transmit/receive the radar signal by using an ultrawide band (UWB) double-ridged horn antenna [Geozondas GZ0126DRH] that was used in the monostatic configuration. The antenna frequency range is $1-26 \mathrm{GHz}$. Beam width of the antenna at $\mathrm{E}$ plane is $37.7^{\circ}$ at $1 \mathrm{GHz}$ and $22.4^{\circ} 8 \mathrm{GHz}$. A turn table with automation software was employed to get different look angle profiles of the tree-body. Also, a PC has to be used 


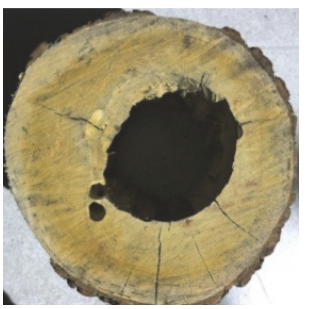

(a)

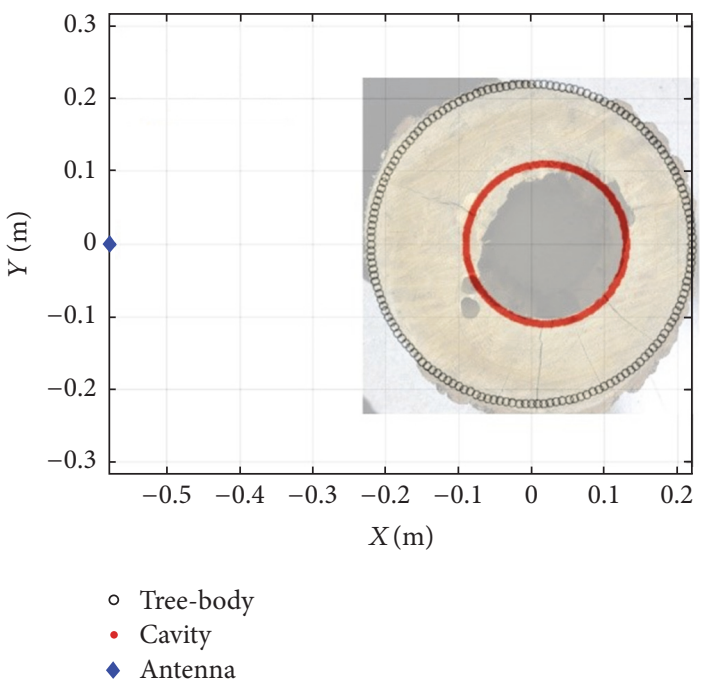

(b)

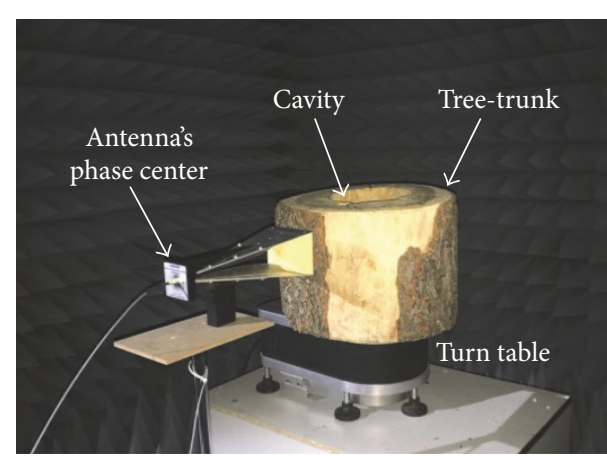

(c)

FIgURE 5: (a) Picture of the piece of pine tree trunk, (b) simulation scenario for the experiment, and (c) picture from the real TIIR experiment.

to synchronously control the VNA and the turn table with a MATLAB [27] script.

For the presented configuration in Figure 4, a piece of pine tree trunk with outer-diameter of approximately $42 \mathrm{~cm}$ was used as the target to be imaged. The tree has a cavity whose center is about $2 \mathrm{~cm}$ away from the center of the tree with a radius of about $11 \mathrm{~cm}$ as pictured in Figure 5(a). Before starting the experiment, we first constructed an approximate $2 \mathrm{D}$ simulation of the measurement that we were going to do to visualize the results out of the experiment and to compare it to real experiment that we were going to conduct later on. In Figure 5(b), the simulation for the experiment was constructed again in MATLAB. Our antenna is assumed to be $58 \mathrm{~cm}$ away from the center of the tree or $36 \mathrm{~cm}$ away from the body of the tree trunk. The geometry for the tree trunk and the cavity was constructed in accordance with the real values as illustrated in Figure 5(b).

For the simulation, the frequency is altered from 1 to $8 \mathrm{GHz}$ for a total of 100 discrete frequencies while the look angle was also varied from $1^{\circ}$ to $360^{\circ}$ for a total of 361 distinct aspects. The tree-body's frequency-independent reflectivity amplitude was chosen to be "1" while that of cavity was selected as "0.1" to emulate the attenuation of EM wave while travelling inside the tree-body. The $2 \mathrm{D}$ range-angle data after running the simulation is presented in Figure 6(a) where we can observe different scattering mechanisms easily: the reflection from the tree-body showed up as a straight line around $36 \mathrm{~cm}$ from the antenna as expected. The front point and the back point of the cavity experienced two sinusoids as the look angle varies along the whole azimuth. Of course, the reflection magnitude for the back point of the cavity is less than that of the front point of the cavity due to attenuation of the EM wave with distance. After applying our sinusoidal template-based focusing algorithm, the TIIR image of this simulation for the experiment was gathered as plotted in Figure 6(b). By comparing this figure with the simulation geometry in Figure 5(b), one can easily see that the suggested algorithm successfully forms the TIIR image of the scenario with good fidelity.

As the final study, we have conducted a real throughthe-tree-imaging-radar experiment as pictured in Figure 5(c). The experiment was performed with the measurement testbed at our anechoic chamber of MEATRC facility. During this experiment, the tree trunk whose picture is seen in Figure 5(a) was put on the turn table. The antenna in monostatic configuration was positioned as seen in Figure 5(c) such that the phase center of the measurement set-up is about $58 \mathrm{~cm}$ away from the scanning axis of the tree trunk just as similar to the simulation. By using the automation software that was adopted by our MATLAB script, turn table has been turned for the full azimuth coverage of $360^{\circ}$ at $1^{\circ}$ increments. For each look angle measurement, the frequency of the VNA was changed from 1 to $8 \mathrm{GHz}$ for a total of 100 discrete frequency points as in the case of the simulation. Therefore, a $2 \mathrm{D}$ scattered electric field matrix, $\mathbf{E}^{\mathbf{s}}(k, \phi)$, of $100 \times 360$ measurement points was collected. Theoretical achievable resolution is calculated as $\Delta r=c /\left(2 * B * \sqrt{\epsilon_{r}}\right)=1.66 \mathrm{~cm}$ for a bandwidth of $B=7 \mathrm{GHz}$ and $\epsilon_{r} \cong 1.65$ (relative electric permittivity of tree trunk). Maximum range is $R_{\max }=N *$ $\Delta r=166 \mathrm{~cm}$ for number of the discrete frequency points $N=100$.

After applying the IFT operation along the frequency axis, $2 \mathrm{D}$ range-aspect data, $\mathbf{E}^{\mathbf{s}}(r, \phi)$, were obtained as plotted in Figure 6(c). We can notice the scattering from the tree's body as a wavy line around 36 to $40 \mathrm{~cm}$ since the perimeter of the tree was not a perfect circle. We also notice the scattering from cavity that experiences the same behavior of the tree's body as the look angle varies during the measurement. Although the attenuation of the EM wave inside the tree texture looks more intense than we have anticipated in the simulation, we can still sense the disturbance of the scattering energy due to existence of the cavity around 50 to $70 \mathrm{~cm}$ in range direction. Next, 


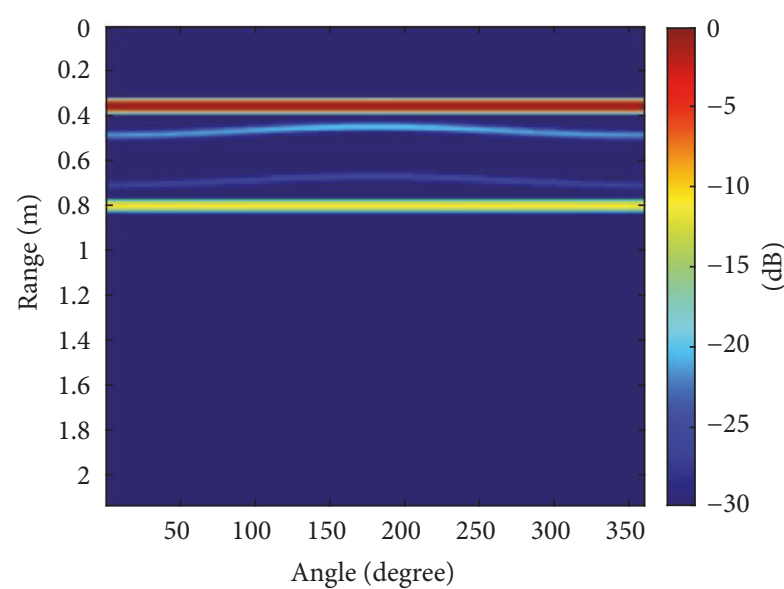

(a)

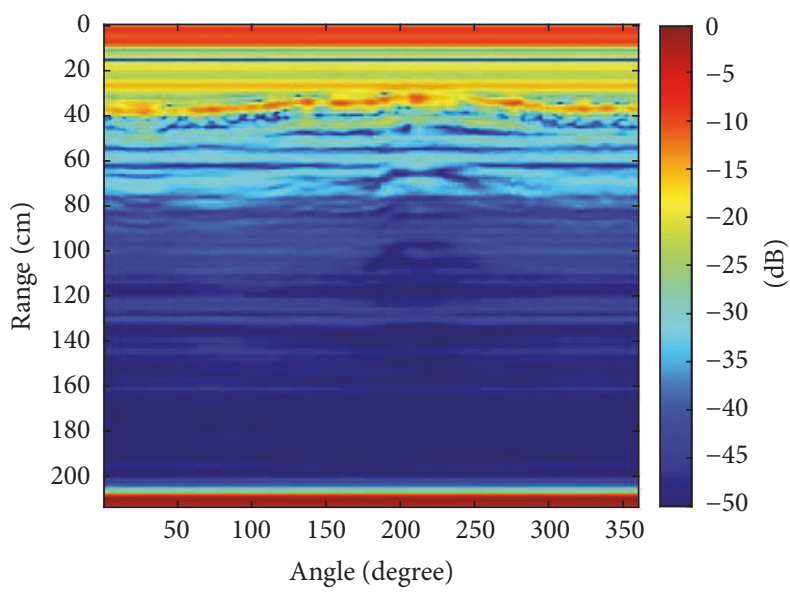

(c)

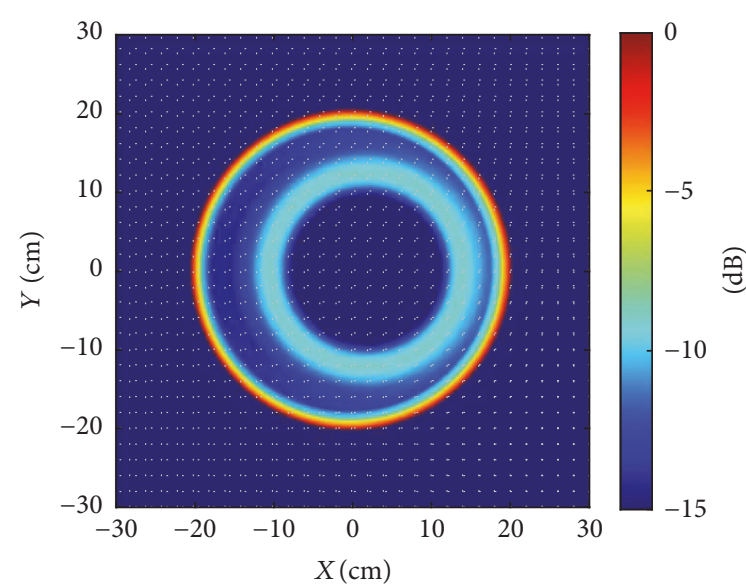

(b)

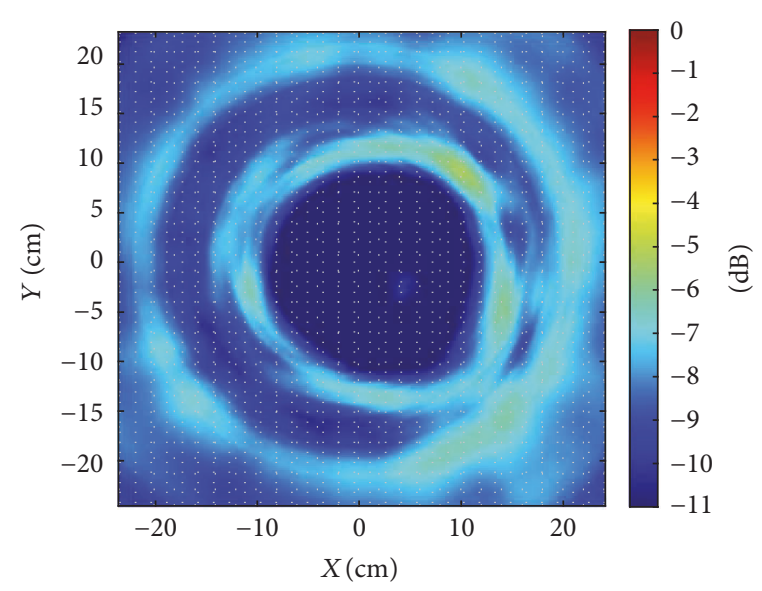

(d)

FIGURE 6: Simulation and measured results for the experiment: (a) simulated range-angle data, (b) simulated TIIR image, (c) measured range-angle data, and (d) measured TIIR image.

we have employed our sinusoidal template based focusing algorithm to migrate the data from range-aspect domain to the image domain such that we have obtained the final TIIR image of the tree trunk as depicted in Figure 6(d). Thanks to the focusing ability of our algorithm, the reflections from the tree-body and the cavity's perimeter have been amplified. Therefore, we can easily notice the borders of impedance changes from air to tree-body and tree-body to cavity and vice versa. Visually comparing the results in Figure 6(d) [measurement] and Figure 6(b) [simulation] with the real picture of tree trunk in Figure 5(a), one can clearly realize that resultant TIIR images successfully form the geometry of treebody and the cavity around their correct locations such that the cavity inside the tree trunk is detected.

\section{Conclusion}

In this work, we have presented our recent work on treeinterior imaging radar research. On this scope, we have introduced a new algorithm based on summation of raw data over sinusoidal templates to be effectively used for TIIR or similar applications. The algorithm is specially developed for multiangle multifrequency monostatic radar set-up with circular scanning geometry of object under test. The details of the algorithm were given together with the assumptions. Then, the algorithm is first tested with simulation data and then a real experiment was conducted at Mersin University's MEATRC facility. Both the simulated and the measured results of TIIR images demonstrate the validity and the success of the proposed algorithm in detecting and localizing the cavity region inside the tree-body.

Algorithm can be modified to include the effect caused by change in the velocity of the EM waves inside the treebody as a future study. This algorithm can also be applied to similar problems such as detecting and imaging breast tumors by employing a very similar geometry of data collection.

\section{Conflicts of Interest}

The authors declare that they have no conflicts of interest. 


\section{Acknowledgments}

This work has been supported by the Scientific and Research Council of Turkey (TUBITAK) under 2211-D Ph.D. scholarship programme and funded by Mersin University Scientific Research Project Unit with Grant no. BAP 2015-TP3-1158. The authors are also thankful to Mersin University MEATRC management for their help and support while conducting the experiments.

\section{References}

[1] J. Fazio, "How to Recognize and Prevent Hazard Trees," Tree City USA Bulletin No. 15, National Arbor Day Foundation, Nebraska City, NE, USA, 1989.

[2] J. Albers and E. Hayes, How to Detect, Assess and Correct Hazard Trees in Recreational Areas, DNR, St. Paul, MN, USA, 1993.

[3] A. Brookes, "Preventing death and serious injury from falling trees and branches," Australian Journal of Outdoor Education, vol. 11, no. 2, pp. 50-59, 2007.

[4] J. Watt and D. J. Ball, "Trees and the risk of harm, report for the national tree safety group," Forestry Commission Rept., Middlesex University, Scotland, 2009.

[5] V. Bucur, Acoustics of Wood, CRC Pres, Boca Raton, FL, USA, 1995.

[6] S. Al Hagrey, "Electrical resistivity imaging of tree trunks," Near Surface Geophysics, vol. 4, no. 12, pp. 177-185, 2006.

[7] G. Catena, L. Palla, and M. Catalano, "Thermal infrared detection of cavities in trees," European Journal of Forest Pathology, vol. 20, no. 4, pp. 201-210, 1990.

[8] A. Franchois, "Microwave permittivity measurements of two conifers," IEEE Transactions on Geoscience and Remote Sensing, vol. 36, no. 5, pp. 1384-1395, 1998.

[9] V. Bucur and I. Böhnke, "Factors affecting ultrasonic measurements in solid wood," Ultrasonics, vol. 32, no. 5, pp. 385-390, 1994.

[10] M. Hasegawa, M. Takata, J. Matsumura, and K. Oda, "Effect of wood properties on within-tree variation in ultrasonic wave velocity in softwood," Ultrasonics, vol. 51, no. 3, pp. 296-302, 2011.

[11] H. Berndt, A. P. Schniewind, and G. C. Johnson, "Ultrasonic energy propagation trough wood: where, when, how much," in Proceedings of the Proc. Of 12th Int. Symposium on NDT of Wood, pp. 57-66, Sopron, 2000.

[12] Y. Zhou, "Microwave imaging based on wideband range profiles," Progress in Electromagnetics Research Letters, vol. 19, pp. 57-65, 2010.

[13] G. Lawday, J. A. Dolwin, D. Lonsdale, and J. R Barnett, "Development and use of stress wave meter, to detect the presence of decay in wood blocks," in Proceedings of the Proc. of 12th Int. Symposium on NDT of Wood, pp. 187-196, Sopron, September.

[14] V. Bucur, "Techniques for high resolution imaging of wood structure: A review," Measurement Science and Technology, vol. 14, no. 12, pp. R91-R98, 2003.

[15] B. Yilmaz, C. Ozdemir, and S. Gokkan, "A focusing algorithm for tree-penetrating radar imaging: An experimental study and concept evaluation," in Proceedings of the 2016 17th International Radar Symposium (IRS), pp. 1-4, Krakow, Poland, May 2016.

[16] J. R. Butnor, J. A. Doolittle, L. Kress, S. Cohen, and K. H. Johnsen, "Use of ground-penetrating radar to study tree roots in the southeastern United States," Tree Physiology, vol. 21, no. 17, pp. 1269-1278, 2001.

[17] C. Özdemir, Ş. Demirci, E. Yiǧit, and B. Yilmaz, "A review on migration methods in b-scan ground penetrating radar imaging," Mathematical Problems in Engineering, vol. 2014, Article ID 280738, 2014.

[18] C. Ozdemir, S. Demirci, E. Yigit, and A. Kavak, "A hyperbolic summation method to focus B-scan ground penetrating radar images: An experimental study with a stepped frequency system," Microwave and Optical Technology Letters, vol. 49, no. 3, pp. 671-676, 2007.

[19] B. Yılmaz, Ş. Demirci, E. Yiğit, and C. Özdemir, "An Experimental Study of Through-the-Wall Radar for Life sign Detection," in Proceedings of Progress in Electromagn, Research Symposium (PIERS '13), pp. 1602-1604, 2013.

[20] M. G. Amin, Through-the-Wall Radar Imaging, Taylor and Francis, Bocaraton, FL, USA, 2010.

[21] A. Muqaibel, A. Safaai-Jazi, A. Bayram, A. M. Attiya, and S. M. Riad, "Ultrawideband through-the-wall propagation," IEE Proceedings-Microwaves, Antennas and Propagation, vol. 152, no. 6, pp. 581-588, 2005.

[22] N. J. Redding and G. N. Newsam, "Inverting the Circular Radon Transform," DTSO Research Report DTSO-RR-0211, August 2001.

[23] http://web.hep.uiuc.edu/home/serrede/P435/Lecture_Notes/ Dielectric_Constants.pdf.

[24] C. Özdemi, Inverse Synthetic Aperture Radar Imaging with Matlab Algorithms, John Wiley Sons, Hoboken, NJ, USA, 2012.

[25] R. Bhalla, J. Moore, and H. Ling, "Global scattering center representation of complex targets using the shooting and bouncing ray technique," IEEE Transactions on Antennas and Propagation, vol. 45, no. 12, pp. 1850-1856, 1997.

[26] C. Ozdemir, R. Bhalla, and H. Ling, "A radiation center representation of antenna radiation patterns on a complex platform," IEEE Transactions on Antennas and Propagation, vol. 48, no. 6, pp. 992-1000, 2000.

[27] MATLAB, R2015a, MathWorks Inc., Natick, MA, 2015. 


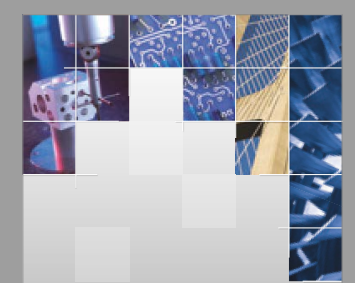

\section{Enfincering}
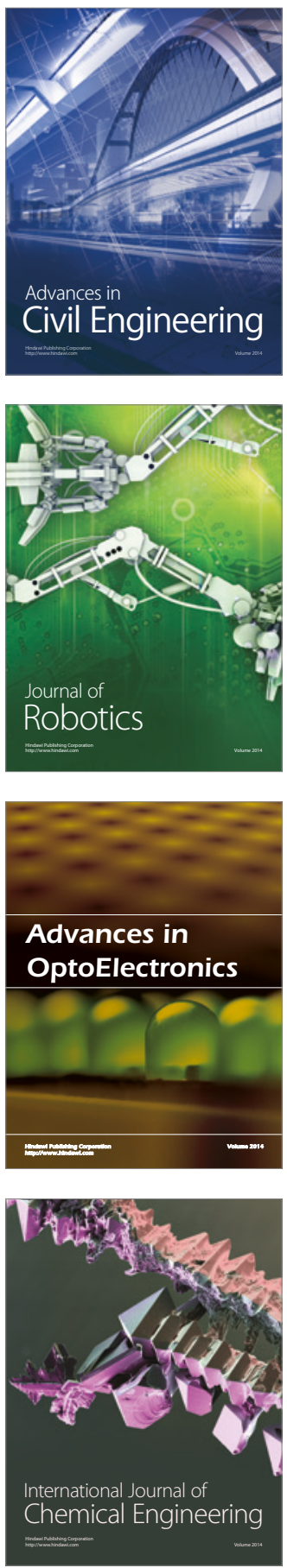

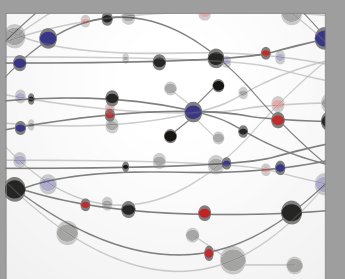

The Scientific World Journal

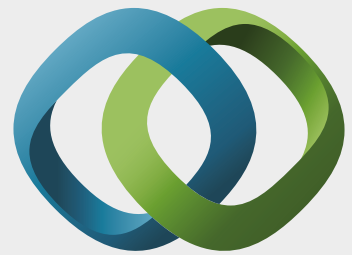

\section{Hindawi}

Submit your manuscripts at

https://www.hindawi.com
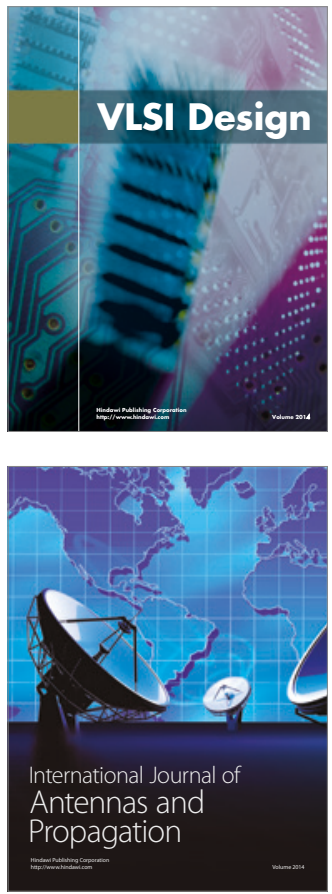

\section{Rotating}

Machinery
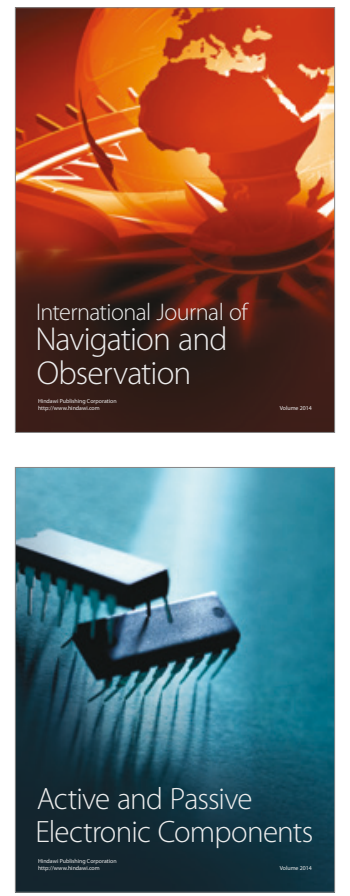
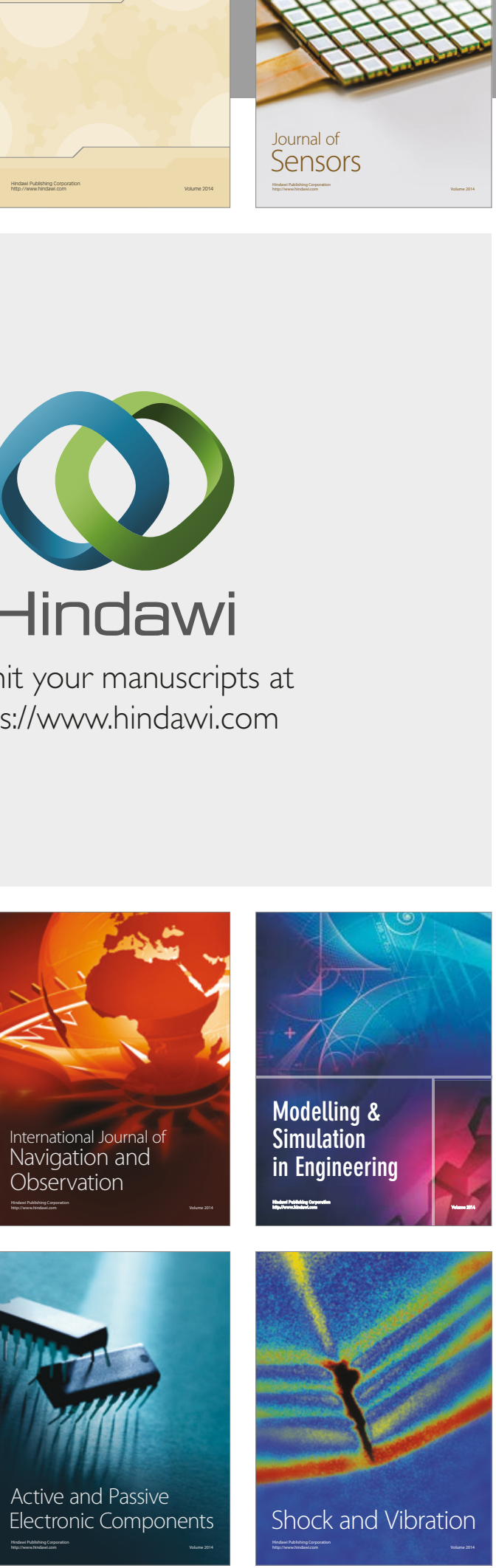
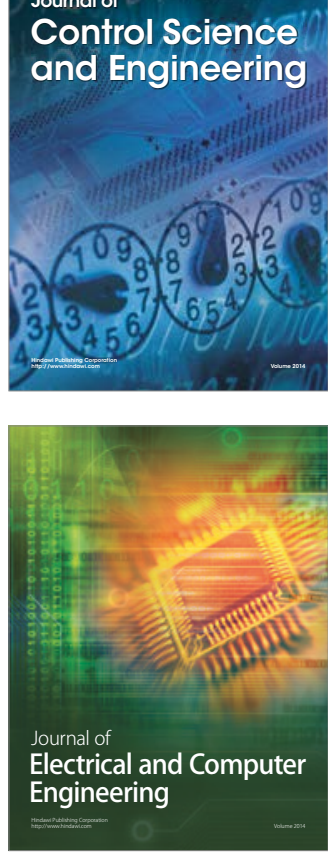

Distributed

Journal of

Control Science

and Engineering
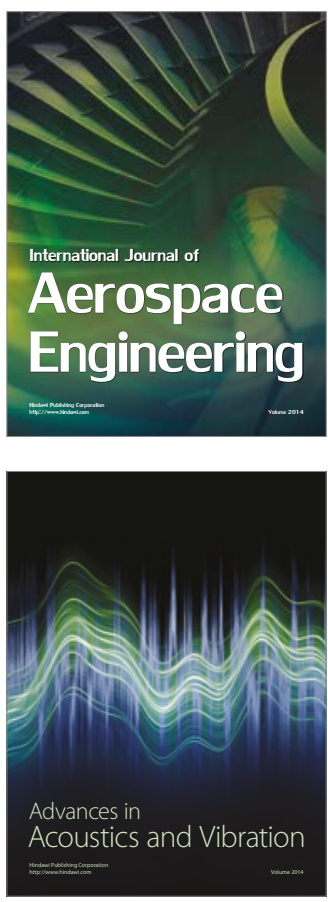

Sensor Networks 